\title{
Characterization and Simulation of Semiconductor Thin Films Using Quantitative Mobility Spectrum Analysis (QMSA)
}

\author{
${ }^{1}$ Nisha Chugh, ${ }^{2}$ A. K. Vishwakarma, ${ }^{3}$ Dr. S. Sitharaman \\ ${ }^{1}$ Asstt. Prof., Department of ECE, Jagannath University, Delhi NCR \\ ${ }^{2}$ Sc. 'B' 'Micro-Bolometer Group', 'MEMS division' SSPL (DRDO), Delhi \\ ${ }^{3}$ Scientist 'F', 'LPE Group' 'IR Division', SSPL (DRDO), Delhi
}

\begin{abstract}
A quantitative mobility spectrum analysis (QMSA) technique of characterizing mobility and concentration of individual carrier species for modern semiconductor hetero-structures by experimentally generated Hall and resistivity data as a function of magnetic field is presented. QMSA enables the conductivity contribution of bulk majority carriers to be separated from that of carriers present at the surface or at the spacer/barrier layer of GaAs-AlGaAs [2] based HEMTs for characterizing carriers developed in '2' DEG region. Beck and Anderson mobility spectrum analysis (MSA) technique was considered as the first trial function. A variation on the iterative procedure of Dziuba and Gorska is used to obtain the mobility spectrum which shows the quantitatively accurate mobility distribution. QMSA is advantageous because in comparison to its previous counter parts such as MCF, MSA and Dziuba \& Gorska; it does not take any prior assumption about the number of electron and hole species as well as their approximate mobilities. A ghost hole along with surface and bulk electron was found in the '2' DEG region developed in HEMTs. In this article, we apply QMSA to both analytical data of $\mathrm{Hg}_{1-x} \mathrm{Cd}_{x} \mathrm{Te}$ and real experimental data of AlGaAs obtained from the Hall measurement system.
\end{abstract}

General Terms: QMSA algorithm, Spline Interpolation, Pinning Point, Jacobi's Iteration, Gauss Seidel Iteration Procedure.

Keywords: Van-der Pauw Technique, Resistivity, Hall Coefficient, HgCdTe, GaAs /AlGaAs, Carrier concentration and mobility, '2' DEG, HEMT.

\section{Introduction}

A compound semiconductor is a compound of elemental semiconductors like $\mathrm{Si}, \mathrm{Ge}, \mathrm{In}, \mathrm{Al}, \mathrm{As}$ and boron etc. from two or more different groups of periodic table. These elements can form binary (e.g. Gallium (III) Arsenide (V), (GaAs)), ternary (e.g. Aluminium Gallium Arsenide (AlGaAs), indium gallium arsenide (InGaAs), Cadmium zinc telluride (CdZnTe), Mercury cadmium telluride ( $\mathrm{HgCdTe}$ ) also called as MCT) and quaternary (e.g. Aluminium Gallium Indium Phosphide (AlGaInP)) alloys. HgCdTe is an alloy of $\mathrm{CdTe}$ and $\mathrm{HgTe}$ and is sometimes claimed to be third semiconductor of technological importance after silicon (IV) and gallium (III) arsenide. Resistivity and Hall coefficient measurements [1] at a single magnetic field are of limited use when mixed conduction systems are implemented, since they provide only averaged values of the carrier concentration and mobility, which do not represent any of the individual species. A detailed information becomes available if the magneto-transport experiments are performed as a function of magnetic field because it is then possible to simultaneously characterize densities and mobilities for each of the multiple electron and hole species. When a compound semiconductor having multi-carriers is grown over another compound semiconductor having multiple carriers, the hetero-structure will contain in total many no of carriers causing mixed-conduction. In order to characterize their transport properties, an analysis with varying large magnetic field than the classic Hall measurement at a single magnetic field is required. Such analysis are generally consisted of a multi-carrier fit (MCF) to the experimental Hall data under the applied magnetic field which is not unique since the starting parameters such as number and type of carriers, and corresponding mobilities and concentrations need to be assumed [3]. Based on all the previous attempts like MCF, MSA [4], Dziuba and Gorska Iterative procedure of obtaining an accurate MS, an approach described by Antoszewski and Faraone et al. [5], and known as quantitative mobility spectrum analysis (QMSA), has been developed and then systematically tested and improved [6-9]. By using the MS of Beck and Anderson as an initial function for a modified Gauss Seidel iterative procedure, the inherent instability of previous iterative procedures during the convergence process is removed and a good fit to the experimental data is obtained. QMSA gave a perfect fit for 2 carriers in the analytical data of $\mathrm{Hg}_{1-\mathrm{x}} \mathrm{Cd} \mathrm{x}$ Te. In this paper, we present and discuss the performance of QMSA for analytical data of $\mathrm{Hg}_{1-\mathrm{x}} \mathrm{Cd}_{\mathrm{x}} \mathrm{Te}$ and for evaluating mobility spectra of carriers developed in ' 2 ' $\mathrm{DEG}$ region of GaAs/AlGaAs hetero-structure HEMTs. 


\section{Van-Der Pauw Technique}

The method was first propounded by Leo J. Van-der Pauw in 1958 [1]. Its power lies in its ability to accurately measure the properties of a sample of any arbitrary shape, so long as the sample is approximately two-dimensional (i.e. it is much thinner than it is wide) and the electrodes are placed on its perimeter. From the measurements made, the following properties of the material can be calculated:

- The resistivity of the material, $\rho$

- $\quad$ The doping type (i.e. P-type or N-type )

- $\quad$ The sheet carrier density of the majority carrier (the number of majority carriers per unit area)

- The mobility of the majority carriers, $\mu$

\subsection{Conditions and Sample Preparation}

In order to use the Van-der Pauw method, the sample thickness must be much less than the width and length of the sample. In order to reduce errors in the calculations, it is preferable to take the sample as a symmetrical one i.e. uniform all across. There are five conditions for using Van-der Pauw technique:

- $\quad$ The sample must have a flat shape of uniform thick-ness all around.

- $\quad$ The sample must not have any isolated holes.

- $\quad$ The sample must be homogeneous and isotropic.

- $\quad$ All four contacts must be located at the edges/corners of the sample.

- The area of contact of any individual contact should be at least an order of magnitude smaller than the area of the entire sample.

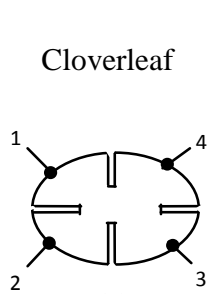

(a)

Square or Square or Rectangle:

Rectangle: Contacts Contacts at the edges at the corners or inside the perimeter

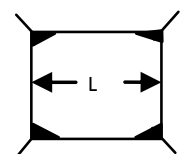

(b)

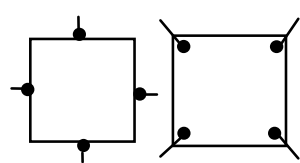

(c)

Preferred

Not Recommended

Figure 1. Some possible contact placements in VDP

Figure 1 above shows the possible contact placement configurations in the VdP technique. The measurements require that four ohmic contacts be placed on the sample.

\section{Mixed-Conduction Multi-Carrier Systems}

In a multi-carrier system having more than one type of carriers, a conventional hall measurement technique provides resistivity $\rho(B)$ and hall co-efficient, $R_{H}(B)$. Hall measurement system directly determines $\mathrm{R}_{\mathrm{xx}}$ and $\mathrm{R}_{\mathrm{xy}}$, (magneto-transport resistances) in longitudinal and transverse direction which ultimately calculate $\rho(B)$ and $R_{H}(B)$ as formalized in eq.(1)

$$
\begin{aligned}
& \rho^{\exp }(\mathrm{B})=1.13 \times \mathrm{f} \times \mathrm{t} \times \mathrm{R}_{\mathrm{xx}} \Omega-\mathrm{cm} \\
& \mathrm{R}_{\mathrm{H}}^{\exp }(\mathrm{B})=\frac{\mathrm{V}_{\mathrm{H}} \mathrm{t}}{\mathrm{IB}} \times 2.5 \times 10^{7} \mathrm{~cm}^{3} / \mathrm{C}
\end{aligned}
$$

Where $\frac{V_{H}}{I}=R_{x y}$, tensor component of resistance in ohms, $t=100 \AA\left(100 \times 10^{-8}\right)$ is the thickness of the AlGaAs epi layer grown on GaAs buffer layer in our experiment, $\mathrm{f}$ is the form factor ( $\mathrm{f}=1$, for perfect geometry), $\mathrm{B}$ is the magnetic field applied along $\mathrm{z}$ axis in units of Gauss (where 1 Tesla $=10^{4}$ Gauss). So finally $R_{H}(B)$ becomes as given in eq. (2) ahead

$$
\mathrm{R}_{\mathrm{H}}^{\exp }(\mathrm{B})=\frac{\mathrm{R}_{\mathrm{xy}} \mathrm{t}}{\mathrm{B}} \times 2.5 \times 10^{7} \mathrm{~cm}^{3} / \mathrm{C}
$$

The diagonal $\left(\sigma_{\mathrm{xx}}\right)$ and hall $\left(\sigma_{\mathrm{xy}}\right)$ conductivity tensor components in the analytical data of a multi-carrier system can be expressed as a sum over the $\mathrm{m}$-species ( $\mathrm{m}$ stands for multi-carriers) in the system as shown in eq. (3)

$$
\sigma_{x x}(B)=\sum_{i=1}^{m} \frac{\mathrm{en}_{\mathrm{i}} \mu_{\mathrm{i}}}{1+\left(\mu_{\mathrm{i}} \mathrm{B}\right)^{2}}
$$




$$
\sigma_{x x}(B)=\sum_{i=1}^{m} S_{i} \frac{e n_{i} \mu_{i}^{2} B}{1+\left(\mu_{i} B\right)^{2}}
$$

Where $\mathrm{n}_{\mathrm{i}}$ and $\mu_{\mathrm{i}}$ are the concentration and mobility of the $\mathrm{i}^{\text {th }}$ carrier species, respectively, and $\mathrm{S}_{\mathrm{i}}$ is +1 for holes and -1 for electrons. The diagonal $\left(\sigma_{\mathrm{xx}}\right)$ and hall $\left(\sigma_{\mathrm{xy}}\right)$ conductivity tensor components for the experimental data will be calculated by experimental resistivity and hall co-efficient values calculated by hall measurement, as given in eq. (4)

$$
\begin{gathered}
\sigma_{x x}(B)=\frac{1}{\rho^{\exp }(B)\left[\left(\frac{R_{H}^{\exp }(B) B}{\rho^{\exp }(B)}\right)^{2}+1\right]} \\
\sigma_{x y}(B)=\frac{R_{H}^{\exp }(B) B}{\rho^{\exp ^{2}}(B)\left[\left(\frac{R_{H}^{\exp }(B) B}{\rho^{\exp }(B)}\right)^{2}+1\right]} .
\end{gathered}
$$

Eq. (3) is modified in the discrete mobility form and is termed as Dziuba and Gorska Jacobi iterative procedure and is given in eq. (5)

$$
\begin{gathered}
\sigma_{x x}\left(B_{j}\right)=\sum_{i=1}^{m} \frac{\left[s^{p}\left(\mu_{i}\right)+s^{n}\left(\mu_{i}\right] \Delta \mu_{i}\right.}{1+\mu_{i}^{2} B_{j}^{2}}=\sum_{i=1}^{m} \frac{S_{i}^{x x} \Delta \mu_{i}}{1+\mu_{i}^{2} B_{j}^{2}} \\
\sigma_{x y}\left(B_{j}\right)=\sum_{i=1}^{m} \frac{\left[s^{p}\left(\mu_{i}\right)-s^{n}\left(\mu_{i}\right] \mu_{i} B_{j} \Delta \mu_{i}\right.}{1+\mu_{i}^{2} B_{j}^{2}}=\sum_{i=1}^{m} \frac{S_{i}^{x y} \mu_{i} B_{j} \Delta \mu_{i}}{1+\mu_{i}^{2} B_{j}^{2}} \ldots
\end{gathered}
$$

Where parameter $\mathrm{m}$, now defines the number of points in the final mobility spectrum not the number of carrier species as in eq. (1) and $B_{i}$ is the spline interpolated experimentally applied varying magnetic field. The ultimate goal is to calculate conductivity density functions $s^{\mathrm{p}}(\mu)\left[\right.$ where $\left.\mathrm{s}^{\mathrm{p}}(\mu)=\operatorname{ep}(\mu) \mu\right]$ and $\mathrm{s}^{\mathrm{n}}(\mu)[$ where $\left.\mathrm{s}^{\mathrm{P}}(\mu)=e \mathrm{e}(\mu) \mu\right]$ which represent the mobility spectra of individual carrier species present in the sample, where $\mathrm{p}(\mu)$ and $\mathrm{n}(\mu)$ are the hole and electron concentration density functions.

\section{QMSA Implementation And Results}

The starting point for the QMSA procedure is to allow for the existence of mobility distribution of hole-like and electron-like species in the semiconductor material. The QMSA uses the Mobility spectrum of Beck \& Anderson [4] in which experimental conductivity tensor vs. magnetic field data is transformed into a continuous profile of carrier mobilities present in the sample as first trial function in order to solve eq. (5) through Gauss Seidel iterative procedure. The ultimate goal is to characterize [10] the semiconductor materials causing mixed conduction so that the mobility spectra of individual carrier species can be known. Convergence coefficients $\mathrm{w}_{\mathrm{x}}=0.03$ and $\mathrm{w}_{\mathrm{y}}=0.003$ minimize the error, speed up the rate of convergence and yield a stable, quantitatively accurate solution, as compared to having $\mathrm{w}_{\mathrm{x}}=\mathrm{w}_{\mathrm{y}}=1$ in the standard iteration procedure. We use 100 mobility points $\mu_{\mathrm{i}}$ per decade, and extend the mobility range considered by Dziuba and Gorska by more than an order of magnitude in each direction by interpolating at low magnetic fields to the data including $\mathrm{B}=0$, and by extrapolating the experimental data at high magnetic fields. In order to verify the ability of QMSA to transform experimental Hall data in the magnetic field domain to mobility domain to have meaningful mobility spectra, synthetic data set of two carriers with their carrier concentration and mobility have been generated by substituting synthetic values of the parameters in eq. (3) and (5). In order to simulate experimental conditions, a $1 \%$ random error is then superimposed onto the synthetic data. The maximum magnetic field is taken to be $8 \mathrm{~T}$ (0.1 to 8Tesla), which is typical for electromagnets used in Hall systems. An example of $n-\mathrm{Hg}_{1-\mathrm{x}} \mathrm{Cd}_{\mathrm{x}} \mathrm{Te}$ conductivity vs. magnetic field at $\mathrm{T}=80 \mathrm{~K}$ is shown in figure 2 (a) and mobility spectra at $\mathrm{T}=80 \mathrm{k}$ and $110 \mathrm{k}$ is shown in figure 2(b) below: 


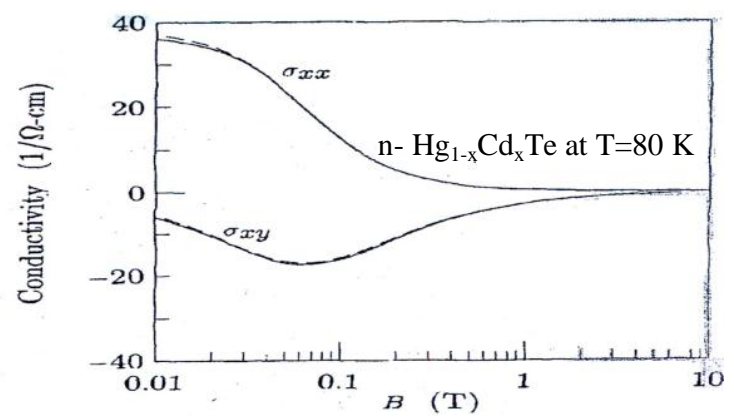

(a)

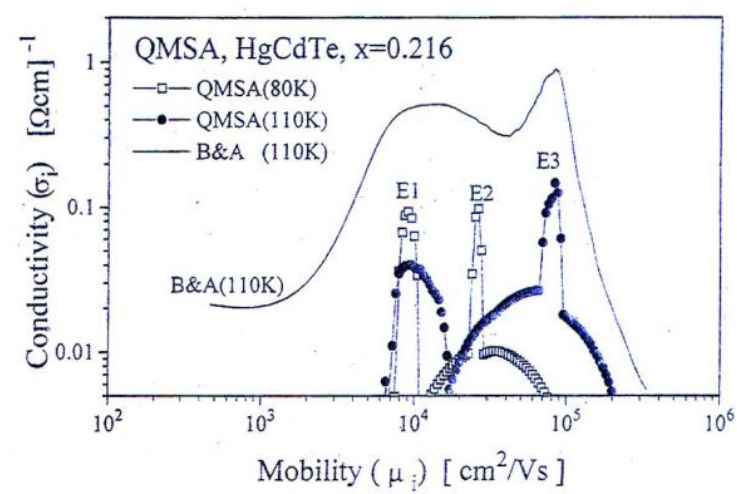

(b)

Figure 2. QMSA for n- HgCdTe: (a) QMSA fit (dashed curves) to the experimental diagonal and Hall conductivities (solid curves) vs. magnetic field for an n-type sample $(x=0.224)$ at $80 \mathrm{~K}[6]$, (b) Mobility spectrum (QMSA) for LPE HgCdTe at 80 and $150 \mathrm{~K}$ and B \& A envelope at $110 \mathrm{~K}$ [5]

Figure 2 (b) shows three electrons E1, E2 and E3 in the mobility spectra. The $80 \mathrm{~K}$ spectrum displays two sharp peaks (E1 and E2), while the $110 \mathrm{~K}$ spectrum contains two peaks (E1 and E3) plus a broad shoulder. The solid line indicated as B \& A is the initial, discrete form of the Beck and Anderson envelope for the iterative procedure.

\subsection{QMSA for Analytical Data-Results}

In the analytical data for two carriers of $\mathrm{n}-\mathrm{HgCdTe}$, carrier concentration and mobility are given as below in the table 1 .

Table 1. Input Values In An Analytical QMSA For HgCdTe

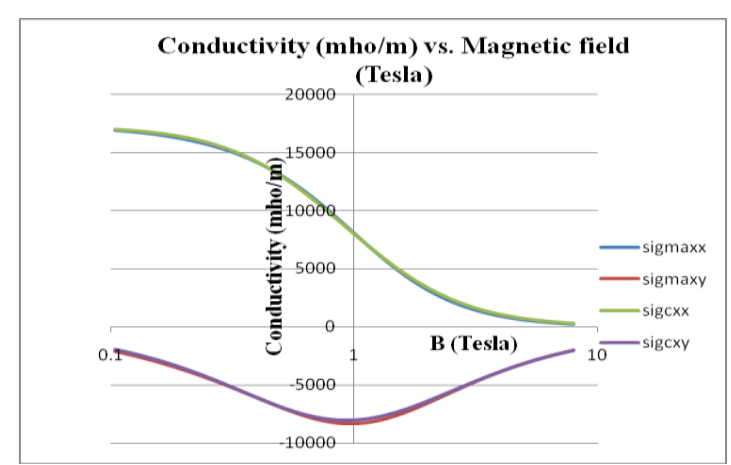

\begin{tabular}{|c|c|c|}
\hline Sr. No. & $\begin{array}{c}\text { Carrier } \\
\text { Concentration, } \mathbf{n} \\
\left(/ \mathbf{m}^{\mathbf{3}}\right)\end{array}$ & $\begin{array}{c}\text { Mobility, } \boldsymbol{\mu} \\
\left(\mathbf{m}^{\mathbf{2}} / \text { volt-sec }\right)\end{array}$ \\
\hline 1. & $-1 \times 10^{23}$ & $1 \mathrm{~m}^{2} /$ volt-sec \\
\hline 2. & $-2 \times 10^{21}$ & $-4 \mathrm{~m}^{2} /$ volt-sec \\
\hline
\end{tabular}

(a) 


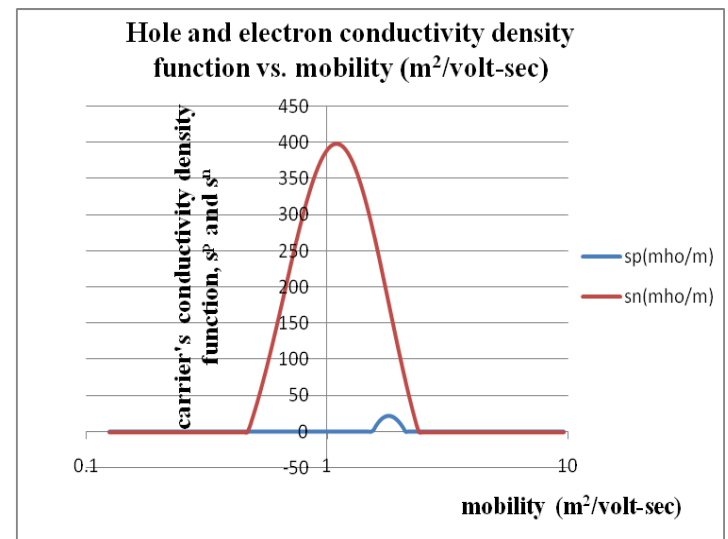

(b)

Figure 3. Graphs of Analytical results of $\mathrm{Hg}_{1-\mathrm{x}} \mathrm{Cd} \mathrm{d}_{\mathrm{x}} \mathrm{Te}$ (where $\mathrm{x}=0.286$ ) for two carriers: (a) Analytically (sigmaxx and sigmaxy) and theoretically (sigcxx and sigcxy) calculated conductivity components with magnetic field, (b) Simulated and QMSA generated mobility spectrum of HgCdTe (Hole and electron density function with mobility)

As shown in figure 3(a) the analytically (shown by blue and red curves) and theoretically (shown by green and violet curves) calculated conductivity components are overlapping with each other giving a verification of QMSA and figure 3 (b) shows the mobility spectra of two carriers given as input in Table 1.

\subsection{QMSA For Experimental Data-Results}

In the '2' DEG region of the HEMT structure QMSA is applied at low temperature of liquid nitrogen, as is the requirement of hall measurement system. $\mathrm{HgCdTe}$ and AlGaAs structures are grown over CZT and GaAs substrates at cryogenic conditions. Liquid nitrogen is the needful temperature requirement material having its own temperature at $77 \mathrm{~K}$. Liquid Helium (He) is also used for extremely low temperature conditions having its own temperature around $4 \mathrm{~K}$. This is the property of the material to be operated under hall measurement at different temperatures, usually ultra-low temperature range.

\subsubsection{GaAs/AlGaAs hetero-structure HEMTs}

To allow conduction, semiconductors are doped with impurities which donate mobile electrons (or holes). However, these electrons are slowed down through collisions with the impurities (dopants) used to generate them in the first place. HEMTs as shown in Figure 3 avoid this through the use of high mobility electrons generated using the hetero-junction of a highly-doped wide-bandgap n-type donor-spacer layer $(\mathrm{AlGaAs})$ and an undoped narrow-bandgap buffer layer with no dopant impurities (GaAs in this case). Si being the tetravalent element is used as a dopant with $\mathrm{Ga}$ and As to form a lattice.

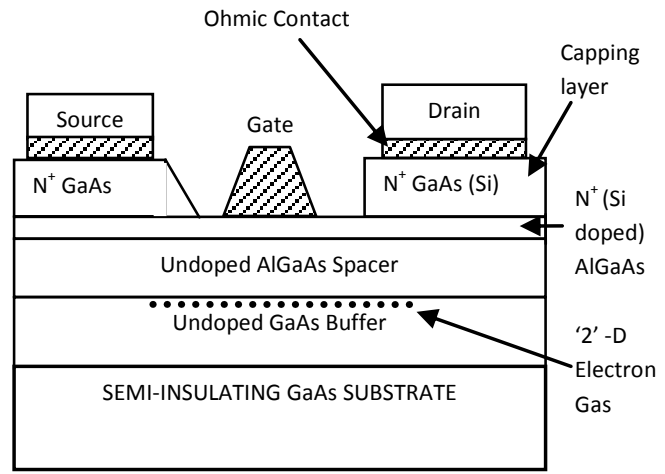

(a) 


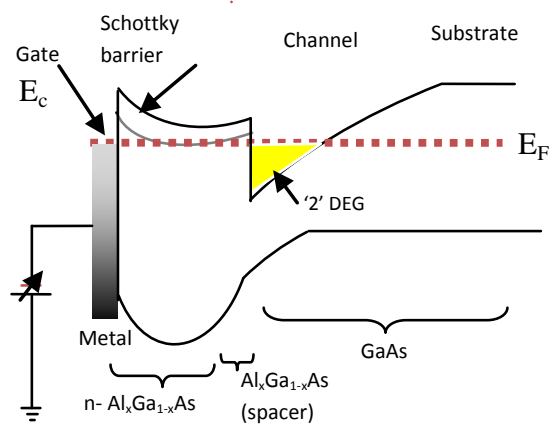

(b)

Figure 4. Experimental Structure (a) GaAs/AIGaAs high-electron-mobility transistor (HEMT) (b) Band gap structure and '2' DEG formation in the quantum well

The thickness of the doped AlGaAs layer grown over undoped GaAs [2] buffer layer is of the order of $100 \AA$ $(\mathrm{t}=100 \AA)$. The $\mathrm{n}$-type AlGaAs layer of the HEMT is depleted completely through two depletion mechanisms:

- $\quad$ Trapping of free electrons by surface states causes the surface depletion.

- Transfer of electrons into the undoped GaAs layer brings about the interface depletion.

The Fermi energy level of the gate metal is matched to the pinning point, which is $1.2 \mathrm{eV}$ below the conduction band. With the reduced AlGaAs layer thickness, the electrons supplied by donors in the AlGaAs layer are insufficient to enter the layer. As a result, bending of the band is moving upward and the two-dimensional electron gas does not appear. When a positive voltage greater than the threshold voltage is applied to the gate, electrons accumulate at the interface and form a two-dimensional electron gas.

\subsubsection{Experimental QMSA for AlGaAs at 77K}

The QMSA was experimentally performed on AlGaAs using liquid nitrogen having its own temperature as $\mathrm{T}=77 \mathrm{~K}$.

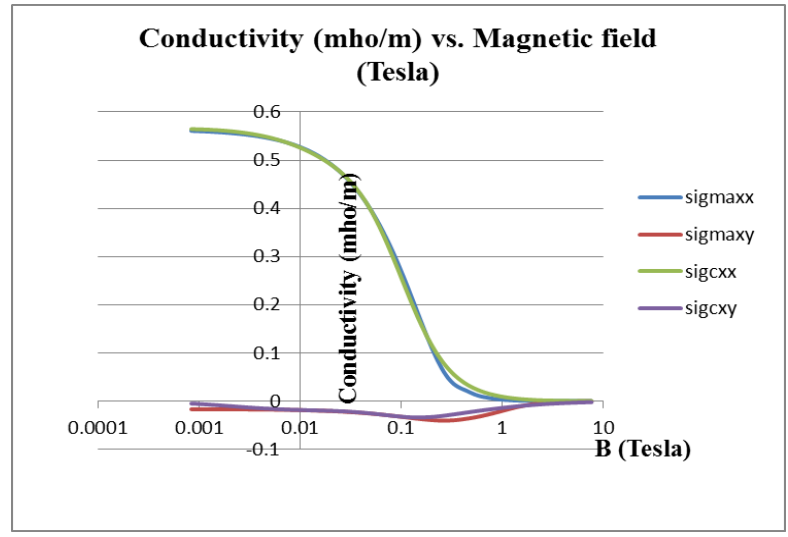

(a)

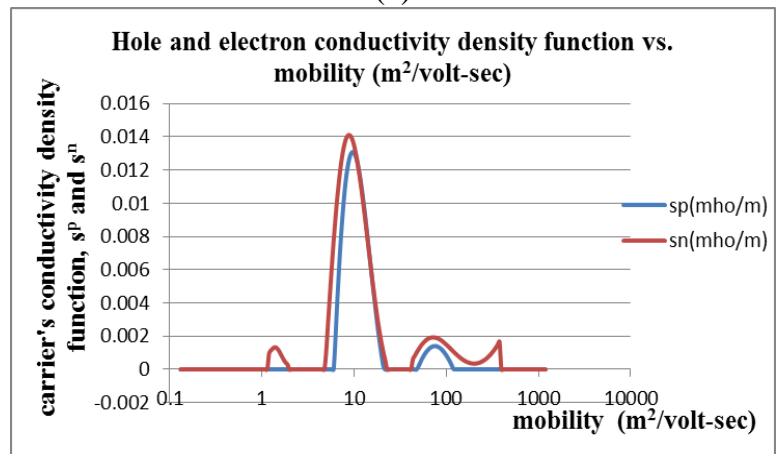

(b)

Figure 5 Graphs of Experimental results of AlGaAs: (a) Experimentally (sigmaxx and sigmaxy) and theoretically (sigcxx and sigcxy) calculated conductivity components with magnetic field, (b) Simulated and QMSA generated mobility spectrum of '2' DEG HEMT (Hole and electron density function with mobility) 
In above figure 5 (a) the experimentally (shown by blue and red curves) and theoretically (shown by green and violet curves) calculated conductivity tensor components are overlapping with each other showing that experimentally calculated and Gauss Seidel iterated values of conductivity components are same. Figure 4 (b) shows the mobility spectra of carriers developed in '2' DEG region of HEMT where a ghost hole was found. The ' 2 ' electrons of mobility approximately ' 1 ' $\mathrm{m}^{2} / \mathrm{volt}-\mathrm{sec}$ and ' 10 ' $\mathrm{m}^{2} / \mathrm{volt}-\mathrm{sec}$ and carrier density $6.25 \times 10^{18} / \mathrm{m}^{3}$ and $8.75 \times 10^{16} / \mathrm{m}^{3}$ are present in the ' 2 ' DEG region as shown by the outer (red) curve in the graph. A ghost hole of mobility $10 \mathrm{~m}^{2} /$ volt-sec and carrier density $8.125 \times 10^{16} / \mathrm{m}^{3}$ is present in ' 2 ' DEG region of HEMT as shown by inner (blue) curve. The curves at and after mobility of $100 \mathrm{~m}^{2} /$ volt-sec are virtual and this much of mobility can't exist for any carriers, even electrons.

\section{Conclusions}

The extensive testing of the QMSA algorithm for analytical data first has shown it to be an extremely accurate, reliable, and convenient technique for analyzing magnetic field-dependent experimental data of $\mathrm{GaAs} / \mathrm{AlGaAs}$ hetero-structure. QMSA is ideally suited for the routine electrical characterization of different wideband gap semiconductor materials and devices, particularly IR materials such as $\mathrm{HgCdTe}$. MCT is a material whose magneto-transport properties tend to be strongly influenced by mixed-conduction of multicarriers i.e. conduction by holes, light holes and electrons as carriers and are well separated by QMSA. For $\mathrm{GaAs} / \mathrm{AlGaAs}$ hetero-structure high doping of $\mathrm{Si}$ as donor impurities is done to make $\mathrm{N}^{+} \mathrm{AlGaAs}$ layer above the undoped $\mathrm{AlGaAs}$ spacer layer and capping layer of GaAs is also highly $\mathrm{N}^{+} \mathrm{Si}$ doped. QMSA spectra of ' 2 'DEG contains electrons as the majority carriers and no minority carriers are seen in the mobility spectra except ghost hole which is approximately same in mobility as the fastest electron in ' 2 ' DEG region. The high mobility carriers developed in HEMTs have their electrical applications in RADARs and communication and optical application in LEDs and Lasers.

\section{Acknowledgments}

The author would like to thank all the scientists and technicians at Solid State Physics Laboratory (SSPL), Defence Research and Development Organization (DRDO) for providing all the facilities pertaining to the experiment, the MBE grown sample of GaAs-AlGaAs and allowed to have practical work there on Hall measurement system.

\section{References}

[1] Van der Pauw, L.J., Feb. 1958, "A method of measuring specific resistivity and Hall Effect of discs of arbitrary shape".

[2] J. W. Allen, Nature, vol. 187, Issue-4735, pp. 403-405 1960,"Gallium Arsenide as a semi-insulator".

[3] M.C. Gold and D.A. Nelson, A4, pp. 2040,1986, Journal of Vaccum Science Technology, "Variable magnetic- field Hall effect measurements and analyses of high purity $\mathrm{Hg}$ vacancy (p-type) $\mathrm{HgCdTe}$ ".

[4] W.A. Beck and J.R. Anderson, vol.62, pp.541, 1987, Journal of Applied Physics, "Determination of electrical transport using a novel magnetic field-dependent Hall technique".

[5] J. Antoszewski, D.J. Seymour, L. Faraone, J.R. Meyer, and C.A. Hoffman, Journal of Electronic Materials,vol. 24, pp. 1255, 1995 "Magneto-transport characterization using quantitative mobility spectrum analysis".

[6] J. Antoszewski, J.R. Meyer, C.A. Hoffman, F.J. Bartoli, L. Faraone, S.P. Tobin, P.W. Norton, C.K. Ard, D.J. Reese, L. Colombo, and P.K. Liao, Journal of Electronic Materials, vol. 25, pp.1157, 1996, "Advanced magneto-transport characterization of LPEgrown HgCdTe by QMSA".

[7] J.R. Meyer, C.A. Hoffman, J. Antoszewski, and L. Faraone, Journal of Applied Physics, vol. 81, pp. 709, 1997, “Quantitative mobility spectrum analysis of multicarrier conduction in semiconductors".

[8] J.R. Meyer, C.A. Hoffman, F.J. Bartoli, J. Antoszewski, and L. Faraone, US Patent Nature 5, issue 789, pp. 931, 1998 “Mobility spectrum analysis for magnetic-field dependent Hall and resistivity data".

[9] J. Antoszewski and L. Faraone, , vol.12, No.4, pp. 347-352, 2000 "Quantitative mobility spectrum analysis (QMSA) in multi-layer semiconductor structures", published in 4th International Conference on Solid State Crystals, Opto-electronics review.

[10] Dieter K. Schroder, 779 pages, Jan 2006. "Semiconductor material and Device Characterization", 3rd edition, A John Wile \& Sons, Inc., Publication, IEEE Press, Wiley-Interscience, Arizona State University Tempe. 